awake surgery, could be a more suitable method of monitoring motor function during tumor removal.

Original article Mikuni $\mathrm{N}$ et al. (2007) Clinical significance of preoperative fiber-tracking to preserve the affected pyramidal tracts during the resection of brain tumors in patients with preoperative motor weakness. J Neurol Neurosurg Psychiatry [doi:10.1136/ jnnp.2006.099952]

\section{RLS: a potentially treatable manifestation of diabetic neuropathy}

Reports on the prevalence of restless legs syndrome (RLS) associated with diabetic neuropathy have reached various conclusions. Gemignani et al. have retrospectively studied the prevalence of RLS in a cohort of patients with diabetes, and they report that the features of the associated neuropathy suggest small-fiber involvement in RLS in a subgroup of such patients.

In a group of 99 consecutive patients referred for neuropathy associated with diabetes mellitus, impaired glucose tolerance or impaired fasting glucose, the investigators found that the prevalence of RLS was increased compared with the general population (33.3\% compared with general population estimates of $3.2-11.1 \%$, and that the condition was more common in patients with distal neuropathy than in patients with multiple mononeuropathy. Small-fiber sensory neuropathy was significantly more common in patients with RLS than in patients without RLS (15/33 vs 15/66). RLS has generally been attributed to dopaminergic dysfunction, but the symptoms of this small-fiber subgroup suggested a different pathogenesis. On the basis of these features, neuropathic pain medications were administered to 20 patients with RLS and were effective in 11 cases.

The authors conclude that RLS is a frequent and potentially treatable presentation of diabetic neuropathy, and that it can be associated with small-fiber involvement. They suggest that RLS does not necessarily arise from dopaminergic dysfunction, but could originate at various levels of the nervous system.

Original article Gemignani F et al. (2007) Restless legs syndrome in diabetic neuropathy: a frequent manifestation of small fiber neuropathy. J Peripher Nerv Syst 12: 50-53

\section{Fever following subarachnoid hemorrhage is associated with poor outcome}

Subarachnoid hemorrhage (SAH) is often followed by fever. The rise in brain temperature can lead to ischemic brain injury, hyperemia, exacerbation of cerebral edema, and increased intracranial pressure. A study of 353 patients with SAH has shown that treatment-refractory fever in the period after SAH, and large increases in temperature in particular, might result in poor outcome.

Despite treatment of fever with standard measures including paracetamol and watercirculating cooling blankets, the maximum daily temperature in the 10 days following $\mathrm{SAH}$ was an average of $1.15^{\circ} \mathrm{C}$ above normal (range $0.04-2.74^{\circ} \mathrm{C}$ ). Poor Hunt-Hess grade (severe initial neurological injury) or intraventricular hemorrhage on admission most strongly predicted fever (both $P<0.001$ ); $\mathrm{SAH}$ sum score, large aneurysm size and loss of consciousness at ictus were also important predictors. After adjustment for confounding factors, above-normal temperature was significantly associated with death or severe disability, reduced instrumental activities of daily living score, and cognitive impairment, at 90 days $\left(P \leq 0.02\right.$ for all); each $1^{\circ} \mathrm{C}$ increase above normal in 10-day mean maximum daily temperature was associated with an threefold increase in the likelihood of death or severe disability. Performing the analysis using calculated daily excess above $38.3^{\circ} \mathrm{C}\left(101.0^{\circ} \mathrm{F}\right)$ rather than maximum daily temperature strengthened these associations still further.

Fernandez et al. note that the rise in temperature following SAH seems to correlate with the severity of the initial bleeding event. Whether fever causes neurological injury or is a marker of its severity is unclear. Measures to prevent fever after SAH could be beneficial and should, say the authors, be studied in clinical trials.

Original article Fernandez A et al. (2007) Fever after subarachnoid hemorrhage: risk factors and impact on outcome. Neurology 68: 1013-1019

\section{The symptoms of Rett syndrome could be reversible}

Rett syndrome, a severe delayed-onset autism spectrum disorder, affects at least 1 in every 